\title{
Observation of twist nematic liquid-crystal lines
}

\author{
M. Škarabot and S. Kralja) \\ Jožef Stefan Institute, Jamova 39, 1000 Ljubljana, Slovenia \\ A. Rastegar and Th. Rasing ${ }^{\text {b) }}$ \\ NSRIM Institute, University of Nijmegen, Toernooiveld 1, 6525ED Nijmegen, The Netherlands
}

(Received 22 April 2003; accepted 12 September 2003)

\begin{abstract}
We have observed that a single groove made by an atomic force microscope (AFM) tip in a polyimide layer strongly aligns nematic liquid crystals locally and have used this phenomenon for studying twist nematic lines. We have measured the intensity profile of light transmitted across a single line and the azimuthal surface energy as a function of the spatial separation of grooves. From these measurements, we have determined the azimuthal surface anchoring energy of the AFM structured and of the untreated polyimide. We find that the twist coherence length, which determines the width of TN lines, is approximately proportional to the cell thickness, while the surface anchoring energy can change it for a factor of 2 at maximum. (C) 2003 American Institute of Physics. [DOI: 10.1063/1.1623925]
\end{abstract}

\section{INTRODUCTION}

The physics of surface-induced alignment of liquidcrystal (LC) molecules has attracted considerable interest in the last thirty years because of the key role that the alignment surfaces play in the operation of electrooptic LC devices such as liquid-crystal displays. Among various surface alignment techniques, the rubbing of a polymer-coated surface with a velvet cloth is most commonly used due to its simplicity and reliability. However this method effects the surface rather inhomogeneously. ${ }^{1,2}$ Therefore, only the average effect of rubbing on the LC alignment can be studied and the control over the resulting anchoring characteristics is rather weak.

Recently, Ruetschi et al. ${ }^{3}$ showed that the atomic force microscope (AFM) in contact mode can be used to obtain relatively smooth polymer aligning surfaces with controllable anchoring strengths. ${ }^{4,6}$ This alignment method has already been applied to obtain specific configurations of polymer layers, ${ }^{5}$ LC gratings, ${ }^{6}$ as well as controllable gray scale ${ }^{7}$ and bistability ${ }^{8}$ properties. Recently, this method was also used for studying the mechanism of LC alignment ${ }^{9}$ and was even shown to successful induce alignment on indium-tin oxide substrates. ${ }^{10}$

In this article, we focus on the influence of a single line, made by the AFM stylus in the polymer layer, on the nematic LC ordering and the resulting optical properties, which were studied with a polarizing microscope. The impact of the line separation on the surface anchoring has been analyzed in a twist nematic (TN) cell. The article is organized as follows. In Sec. II, we present the experimental results. The model used to interpret the measurements is described in Sec. III. The results are discussed in Sec. IV and summarized in the last section.

\footnotetext{
a) Also at: Faculty of Education, University of Maribor, Koroška 160, 2000 Maribor, Slovenia.

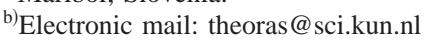

\section{EXPERIMENT}

We used a 5CB (4'-n-pentyl 4-cyanobiphenyl) nematic LC confined in plane-parallel TN cells. In such cells, the twist deformation is imposed by the surface anchoring conditions. The commercial indium-tin oxide glass plates of the cell were coated with a polyimide aligning film of about 100 $\mathrm{nm}$ using the spin coating technique. One glass plate of the cell was rubbed with a soft velvet cloth, while the other was structured with the AFM tip. The anchoring directions of opposite plates were set perpendicular to enforce a twist deformation to the enclosed nematic LC. The thickness $d$ of the cells was around $6 \mu \mathrm{m}$.

A Digital Instruments Dimension 3000 AFM and cantilevers with standard silicon nitride tips with an average tip diameter of $20 \mathrm{~nm}$ were used for scanning the polyimide surface in the contact mode. The scanning force was around $1 \mu \mathrm{N}$. Square patterns with dimensions around $100 \times 100$ $\mu \mathrm{m}^{2}$ were prepared in the normal, i.e., zigzag scan mode. On scanning the polyimide surface, the tip produces grooves by pushing the polymer aside as can be seen in a non-contact AFM image (Fig. 1). The width $w$ of the tip-affected polymer area, tending to align LC molecules along the scanning direction, is approximately $w \sim 30 \mathrm{~nm}$. The remaining area, to which we refer as the surrounding surface, seems to be unchanged. It appears like a typical nonoriented polymer surface in which no direction is singled out on average.

Cells were filled with the LC in the isotropic phase, just above the transition to the nematic phase. The nematic structure was studied using polarizing microscopy. The spatial dependence of the transmitted light through TN patterns was determined from the images taken by a 10-bit digital camera. Figure 2 shows the polarization optical micrographs of two TN patterns. Each pattern consists of several AFM made lines in an area of $100 \mu \mathrm{m}^{2}$, that exhibit well defined TN deformations. Henceforth, we refer to them as TN lines. They appear because of the grooves and the polymer chain alignment created by the AFM tip. ${ }^{9}$ In Fig. 2, we compare the 


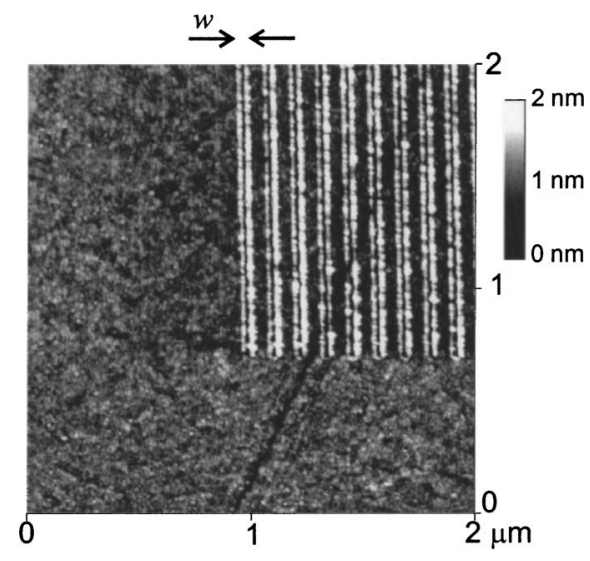

FIG. 1. Tapping mode AFM picture of the polyimide surface after contact mode scanning with the AFM tip with a force of $1 \mu \mathrm{N}$.

effect of the surrounding surface of the AFM scanned plate on the TN lines. In case (a), prior to AFM scanning, this plate was homogeneously rubbed with a soft velvet cloth perpendicular to the AFM scanning direction, while in case (b), it was not. We refer to these two cases as treated (a) and untreated (b), respectively. In case (a), the top plate and the surrounding surface of the AFM structured plate enforce the homogeneous tangential anchoring along their common direction. Therefore, the polarization of the light is not rotated in the surrounding region and light is only transmitted via the TN lines. In case (b), the anchoring of the surrounding surface depends on the history of the sample. We have measured that the intensities and the widths of the TN lines strongly depend on the surrounding surface treatment and we will discuss these phenomena in the last section.

By increasing the number of lines in one square, the extent of the AFM affected area increases as is shown in Fig. 3 . In this case only, the top plate was rubbed with a soft velvet cloth. Since the AFM tip makes zigzag lines in the normal scan mode, we define the line separation $L$ as the maximum line separation at the top or at the bottom side of the TN square. The apparent width of the twist lines on the untreated polymer is around $2 \mu \mathrm{m}$. Consequently, we can observe separate lines under a polarizing microscope down to $L \approx 2.5 \mu \mathrm{m}$.

Using the cell rotation method, ${ }^{11}$ we have also measured the average azimuthal anchoring strength $W_{\phi}$ of the surface, which was treated only with the AFM. Figure 4 shows $W_{\phi}$ of
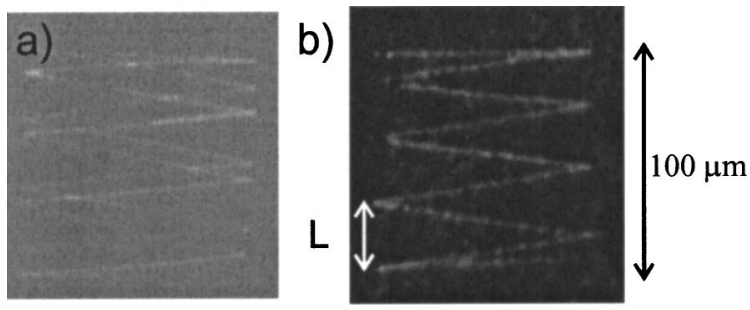

FIG. 2. Images of TN lines under polarizing microscope. Lines are made by the AFM tip on the rubbed polyimide surface (a) and on the untreated polyimide surface (b). Note that image (a) looks completely black under a microscope and the contrast and intensity are increased afterward numerically.

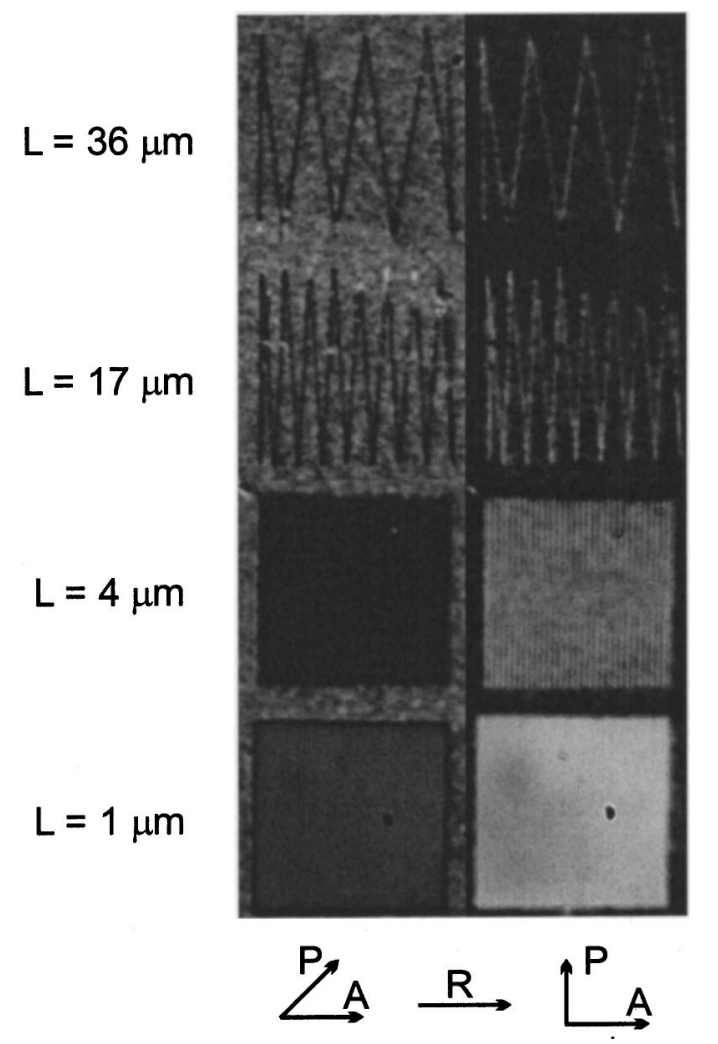

FIG. 3. Polarizing microscope picture of $\mathrm{TN}$ squares with different line separation $L$. P, A, and $\mathrm{R}$ show the direction of polarizer, analyzer, and rubbing direction on the top plate.

the AFM treated surface as a function of $L$. For a strong enough anchoring at the top plate, which is realized in our sample, it roughly holds that $W_{\phi} \propto w / L$ for $w \ll L .{ }^{4} W_{\phi}$ approaches zero for large enough $L$ since no direction is preferred on average for the unoriented polymer surface. With decreasing $L$, the effective anchoring strength first increases proportional to $1 / L$, which was already observed by Wen and Rosenblatt ${ }^{4}$ in a similar system. Additionally, we have observed that $W_{\phi}$ saturates below $L \approx 0.5 \mu \mathrm{m}$ at $W_{\phi}^{s} \approx 1.7$ $\times 10^{-5} \mathrm{~J} / \mathrm{m}^{2}$, which is in agreement with what we observed before. ${ }^{9}$ The fact that $W_{\phi}$ saturates at $L \approx 0.5 \mu \mathrm{m}$, although the width directly affected by the AFM tip is much smaller $(w \approx 30 \mathrm{~nm})$, is a result of the correlation between the LC molecules.

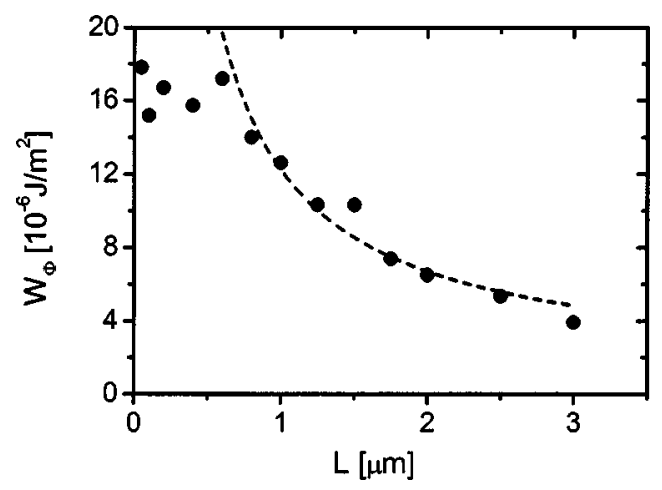

FIG. 4. Azimuthal surface anchoring $W_{\phi}$ as a function of the line separation $L$. Dashed line represents $1 / L$ dependence. 


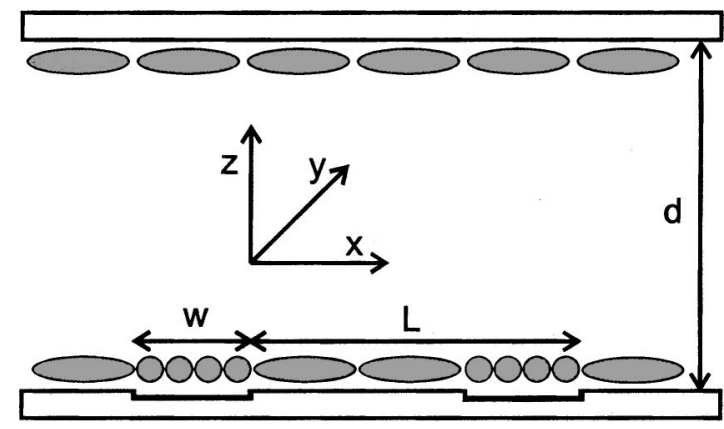

FIG. 5. Schematic representation of the LC orientation on the cell plates.

\section{THEORY}

\section{A. Geometry of the problem and parameterization}

The model system with the essential ingredients for our studies is depicted in Fig. 5. The unit vectors along the $\{x, y, z\}$ axes of the Cartesian coordinate system are labeled with $\mathbf{e}_{x}, \mathbf{e}_{y}$, and $\mathbf{e}_{z}$, respectively. The nematic LC is confined between two plates placed at $z=0$ and $z=d$. Both plates enforce parallel molecular alignment in the following way. The upper plate is rubbed in the $x$-axis direction resulting in a uniaxial ordering along it. The bottom plate exhibits the structured part of the unit, which consists of two regions. In the first region (the twist line surface), with a width $w$, the surface is rubbed with the AFM tip along $\mathbf{e}_{y}$ causing uniaxial ordering in this direction. The second region (the surrounding surface), with a width $L-w$, is either rubbed in the $\mathbf{e}_{x}$ direction or is left untreated. In the treated case, relatively strong uniaxial ordering along $\mathbf{e}_{x}$ is enforced (corresponding to Fig. 5), while the anchoring condition in the untreated case depends on the history of the sample. Because the cell was filled in the isotropic phase, the LC molecules are randomly oriented in the $x y$ plane. However, due to the elastically transmitted influence of the top plate, a relatively weak uniaxial ordering along the $x$ axis is expected to develop on the bottom plate as well.

For a qualitative and quantitative explanation of the measurements, we start from the Landau-de Gennes model in terms of the nematic order parameter tensor $Q$. The uniaxial nematic ordering can be described with ${ }^{12}$

$$
Q=S\left(\mathbf{n} \otimes \mathbf{n}-\frac{1}{3} \underline{I}\right) .
$$

The nematic director field $\mathbf{n}$ points along the local average molecular long axis orientation and the uniaxial order parameter $S$ measures the extent of fluctuations around this direction.

The free energy $F$ of the system consists of volume and surface contributions:

$$
F=\oint \oint \int\left(f_{h}+f_{e}\right) d^{3} \mathbf{r}+\oint \int f_{a} d^{2} \mathbf{r} .
$$

The first integration runs over the LC volume and the second one over the confining surface.

The homogeneous $\left(f_{h}\right)$, elastic $\left(f_{e}\right)$, and surface positional anchoring $\left(f_{a}\right)$ free energy density contributions, expanded in terms of $Q$, are approximately expressed as ${ }^{12,13}$

$$
\begin{aligned}
& f_{h}=A\left(T-T_{*}\right) \operatorname{Tr} \underline{Q}^{2}-B \operatorname{Tr} \underline{Q}^{3}+C\left(\operatorname{Tr} \underline{Q}^{2}\right)^{2}, \\
& f_{e}=L_{n}|\nabla \underline{Q}|^{2}, \\
& f_{a}=\frac{W_{0}}{2} \operatorname{Tr}\left(\underline{Q}-\underline{Q}_{a}\right)^{2},
\end{aligned}
$$

respectively. The quantities $A, B$, and $C$ are material constants, $T$ is the temperature, $T_{*}$ is the supercooling temperature limit, $L_{n}$ is the characteristic bare nematic elastic constant, $W_{0}$ is the anchoring constant, and $\underline{Q}_{a}$ determines the nematic local ordering at the surfaces.

The homogeneous free energy term $f_{h}$ defines the degree of nematic ordering in the homogeneous bulk state. The elastic term $f_{e}$ tends to enforce a spatially homogeneous value of $\underline{Q}$. The surface term $f_{a}$ is minimized for $\underline{Q}=\underline{Q}_{a}$.

For our geometry, the director is in the $x y$ plane and we parameterize the director field as

$$
\mathbf{n}=\mathbf{e}_{x} \cos \theta+\mathbf{e}_{y} \sin \theta,
$$

where $\theta=\theta(x, z)$ only. We further neglect spatial variations in $S$ and assume a homogeneous tangential anchoring condition along the surface imposed easy direction $\mathbf{e}_{a}^{(a)}$ $=\mathbf{e}_{x} \cos \theta_{a}^{(a)}+\mathbf{e}_{y} \sin \theta_{a}^{(a)}$. The superscript (a) stands for rubbed surface (RUB), AFM scanned surface (AFM), and for nontreated surface (NON). The most relevant free energy contributions in this case are

$$
f_{e} \approx \frac{K}{2}\left(\left(\frac{\partial \theta}{\partial x}\right)^{2}+\left(\frac{\partial \theta}{\partial z}\right)^{2}\right), \quad f_{a} \approx \frac{W^{(a)}\left(\theta-\theta_{a}^{(a)}\right)^{2}}{2} .
$$

Here $K=4 L_{n} S^{2}$ is the conventional Frank elastic constant in the single nematic constant approximation and $W^{(a)}$ $\approx W_{0}^{(a)} S^{2}$ is the effective anchoring strength.

The $\theta=\theta(x, z)$ spatial dependence is determined by bulk

$$
\frac{\partial^{2} \theta}{\partial x^{2}}+\frac{\partial^{2} \theta}{\partial z^{2}}=0
$$

and surface

$$
\pm \frac{\partial \theta}{\partial z}+\frac{W^{(a)}}{K}\left[\left(\theta-\theta_{a}^{(a)}\right)=0\right]_{z=0, d}
$$

Euler-Lagrange equations. In Eq. (6b) the - and + sign correspond to $z=0$ and $z=d$, respectively. The easy directions are determined by $\theta_{a}^{(\mathrm{AFM})}=\pi / 2, \theta_{a}^{(\mathrm{RUB})}=\theta_{a}^{(\mathrm{NON})}=0$. The most relevant characteristics lengths entering the model are the geometrical lengths $d, L, w$, and the surface extrapolation lengths $d_{e}^{(a)}=K / W^{(a)}$. In the untreated sample, the uniaxial $\xi_{n} \approx \sqrt{L /\left|A\left(T-T_{*}\right)\right|}$ or the biaxial $\xi_{b} \approx \sqrt{L / B}$ correlation length might be important in case of strong distortions imposed by the surrounding surface.

\section{B. Light transmission}

The Euler-Lagrange equations were solved numerically using the overrelaxation method. ${ }^{14}$ From the obtained director structure, the light transmission intensity profiles were calculated. In the simulation, the birefringent properties of the uniaxial nematic LC were considered. The ordinary and extraordinary components of the linearly polarized incident 
ray propagate with velocities $c_{0}=c / n_{0}$ and $c_{e}\left(\theta_{r}\right)$ $=c / n_{e}\left(\theta_{r}\right)$, respectively. Here $c$ is the velocity of electromagnetic waves in the vacuum, $\theta_{r}$ is the angle between the local optical axis $\mathbf{n}(\mathbf{r})$ and the ray direction, $n_{0}$ is the ordinary and $n_{e}\left(\theta_{r}\right)=n_{0} n_{e} / \sqrt{n_{0}^{2} \sin ^{2} \theta_{r}+n_{e}^{2} \cos ^{2} \theta_{r}}$ is the extraordinary refractive index, $n_{e}=n_{e}(\pi / 2)$. The phase shift between the ordinary and extraordinary component at the exit of the LC sample depends on the director field that the ray experiences on its path. The transmission intensity maps different director patterns providing $L$ is much larger than the wavelength $\lambda$ of the incident light.

In the simulation, the incident ray is along the $\mathbf{e}_{z}$ direction, the analyzer and polarizer are oriented along $\mathbf{e}_{x}$ and $\mathbf{e}_{y}$, respectively. The cell is divided into subcells of small enough width $\Delta z$ so that the refraction indices can be assumed to be spatially homogeneous within them. Their value is determined by the local director field orientation. The total effect on the electric polarization vector is then calculated for the rays transversing the sample. The resulting transmission $T$ at a point $(x, y)$ is calculated as: ${ }^{15}$

$$
T=\left|\mathbf{e}_{A} \cdot \underline{P} \cdot \mathbf{e}_{P}\right|^{2},
$$

where $\underline{P}$ is the transformation matrix, and $\mathbf{e}_{A}, \mathbf{e}_{P}$ are the orientations of the analyzer and polarizer, respectively. The transformation matrix is formally written as

$$
\underline{P}=\prod_{i} \underline{R}_{i} \underline{S}_{i} \underline{R}_{i}^{-1}
$$

The index $i$ runs over the subcells in the $z$ direction. The rotation matrix $\underline{R}_{i}$ performs the transformation of the polarization vector from subcell $\mathrm{i}-1$ to the $i$ th subcell. The matrix $\underline{S}_{i}$ introduces the appropriate phase shift in the $i$ th subcell:

$$
\underline{S}_{i}=\left|\begin{array}{cc}
\exp (i \pi \Delta n \Delta z / \lambda), & 0 \\
0, & \exp (-i \pi \Delta n \Delta z / \lambda)
\end{array}\right|,
$$

and $\Delta n=n_{e}-n_{0}$.

\section{RESULTS AND DISCUSSION}

We first analyze the transmission intensity $(T)$ profile of TN lines shown in Fig. 6 for two different surrounding surface treatments. The orientation of the polarizer and analyzer in our approach yields $T=0$ for a homogeneously aligned nematic LC and $T=1$ for the "ideal" linear nematic twist deformation $\theta(z)=\pi / 2 z / d$.

The results appear to be very sensitive for the surrounding surface treatment. The light transmission intensity is in both samples well below 1 because of a relatively low anchoring strength $W^{(\mathrm{AFM})}$ and the narrowness $(w \ll L)$ of the AFM treated region. In the treated sample [Fig. 6(a)], the maximum transmitted intensity at the center of the line is only $2 \%$. In the calculation, we have used $K=5 \times 1 \theta^{-12} \mathrm{~N}$, $W^{(\mathrm{RUB})}=10^{-4} \mathrm{~J} / \mathrm{m}^{2}, d=6 \mu \mathrm{m}$, and $w=30 \mathrm{~nm}$. We get the best intensity profile approximation with $W^{(\mathrm{AFM})}=2$ $\times 10^{-5} \mathrm{~J} / \mathrm{m}^{2}$. This value is very close to the saturation value at the small line distance separation (Fig. 4). Note that the simulated transmission profile $T(x)$ is narrower than the measured ones. We believe that this is mainly due to diffraction, which was not taken into account in the simulations.

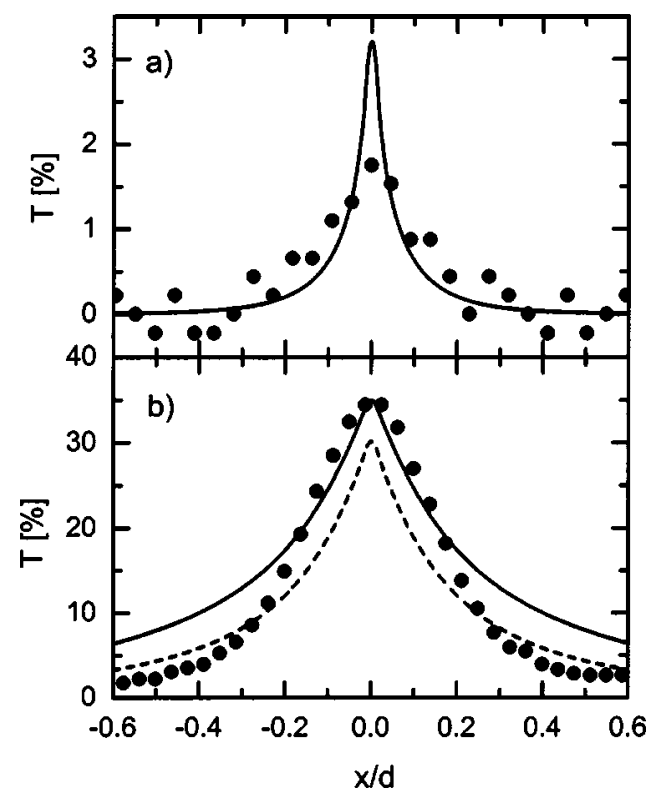

FIG. 6. Measured (O) and calculated (-) intensity profile of the transmitted light through a single $T N$ line made on the rubbed surface (a) and on the untreated surface (b). Parameters in the calculation are: $w=25 \mathrm{~nm}$ (a) and $\quad w=40 \mathrm{~nm} \quad(\mathrm{~b}), \quad W^{(\mathrm{AFM})}=2 \times 10^{-5} \mathrm{~J} / \mathrm{m}^{2}, \quad W^{(\mathrm{RUB})}=10^{-4} \mathrm{~J} / \mathrm{m}^{2}, \quad d$ $=6 \mu \mathrm{m}, K=5 \times 10^{-12} \mathrm{~N}, \lambda=500 \mathrm{~nm}, n_{e}-n_{0}=0.2$, and strong anchoring condition on the top plate. In (b), $W^{(\mathrm{NON})}=10^{-7} \mathrm{~J} / \mathrm{m}^{2}$ (solid line) and $W^{(\mathrm{NON})}=10^{-6} \mathrm{~J} / \mathrm{m}^{2}$ (dashed line). The polarizer and analyzer are set perpendicular.

The width of the region in which the nematic twist deformation is present is namely comparable to $\lambda$. In the untreated sample [Fig. 6(b)] the transmission intensity profiles vary a lot due to the random orientation of LC molecules on the untreated polymer (see Fig. 3). Nevertheless, we can estimate $W^{(\mathrm{NON})}=(1 \pm 1) \cdot 10^{-6} \mathrm{~J} / \mathrm{m}^{2}$.

The calculations also show that the twist angle above the AFM line is less than $90^{\circ}$ since LC molecules at the AFM line deviate from the scanning, i.e., easy direction. In both samples, this deviation is the result of the elastic deformation which is caused by the strong anchoring at the opposite rubbed plate. In the treated sample, the deviation is additionally increased due to the strong anchoring in the surrounding region, which is perpendicular to the AFM line. This effect, that the macroscopic easy direction is the result of two perpendicular neighboring easy directions at the surface, has been observed before by Kim et al. ${ }^{8}$

Next we analyze the influence of the characteristic lengths of the model on the width of the TN lines. For this purpose, we define the coherence distance $\xi_{t}$

$$
\left\langle\theta\left(x=\xi_{t}\right)\right\rangle_{z}=\frac{1}{2}\langle\theta(x=0)\rangle_{z} .
$$

This distance measures the persistence of the nematic twist deformation into the surrounding bulk region. Here $x=0$ defines the center of the TN line. The parentheses $\langle\ldots\rangle_{z}$ indicate the average along the $z$ axis. The ideal linear nematic twist deformation yields $\langle\theta\rangle_{z}=\pi / 4$ and the ideal homogeneous deformation $\langle\theta\rangle_{z}=0$.

In Fig. 7, we show the calculated $\xi_{t}$ as a function of the surface anchoring energy of the surrounding region $W^{(a)}$ for 


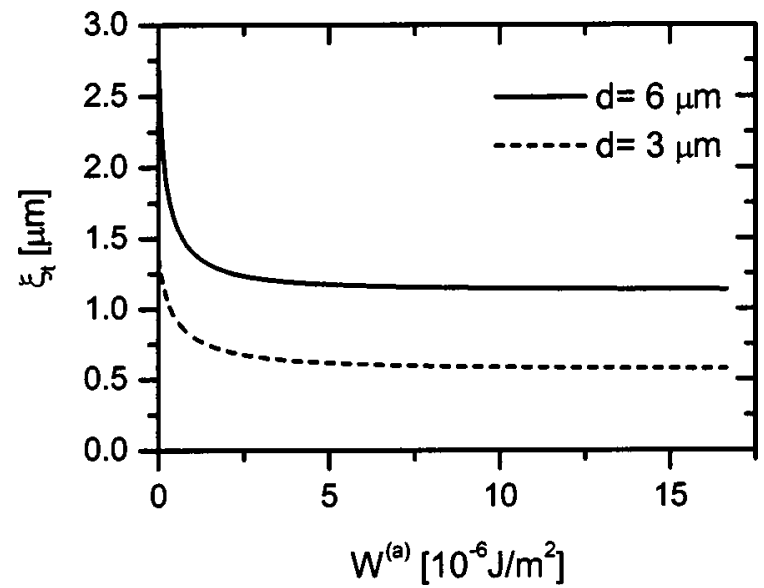

FIG. 7. The twist coherence length $\xi_{t}$ as a function of the surface anchoring energy of the surrounding region $W^{(a)}$ for two different cell thicknesses, $K=5 \times 10^{-12} \mathrm{~N}, W^{(\mathrm{AFM})}=2 \times 10^{-5} \mathrm{~J} / \mathrm{m}^{2}$.

two different cell thickness and for strong anchoring at the top plate. Since the bulk Euler-Lagerange equation does not enforce any characteristic length to the system, the results show that the sample thickness $d$ dominantly influences $\xi_{t}$. Calculations approximately yield $\xi_{t} \propto d$. Additionally, $\xi_{t}$ depends on the surrounding region only for very weak anchoring $\left(W^{(a)}<5 \times 10^{-6} \mathrm{~J} / \mathrm{m}^{2}\right)$. We have measured the width [full width at half maximum (FWHM)] of the TN lines, which is proportional to $\xi_{t}$. From several measurements, we got the average values FWHM $=2.3 \mu \mathrm{m}$ for untreated and FWHM $=1.5 \mu \mathrm{m}$ for the treated case (see Fig. 6). Figure 7 shows that the width of the line is considerably affected by $W^{(a)}$ only for very weak anchoring. Since the width for the untreated case is approximately 50\% larger than the width for the treated case, we can estimate surface anchoring of the untreated polymer to be $W^{(\mathrm{NON})}=(5 \pm 2) \cdot 10^{-7} \mathrm{~J} / \mathrm{m}^{2}$. For the treated (untreated) cases, we thus find $\xi_{t}$ $=1.2 \mu \mathrm{m}(0.8 \mu \mathrm{m})$. Though these values are similar to the ones that were reported before, ${ }^{10}$ the present definition and determination is more precise as it takes the optical properties of the TN lines correctly into account.

We have also tested the influence of the surface induced biaxiality on $\xi_{t}$. Our simulations suggest that biaxiality becomes important when $W^{(a)}>10^{-3} \mathrm{~J} / \mathrm{m}^{2}$ which is not expected to be realized in our samples.

\section{CONCLUSIONS}

We have shown that single AFM made lines on a polyimide surface align nematic LC very well. AFM lines were made on untreated polyimide and on cloth rubbed polyimide perpendicular to the rubbing direction. In both cases, such lines can be observed as TN lines under a polarizing micro- scope. We have measured and analyzed the transmitted light through TN lines. The transmission light intensity and the linewidth strongly depend on the treatment of the surface region that surrounds the twist line. The linewidth (FWHM) and the maximal light transmission intensity was $1.5 \mu \mathrm{m}(2.5$ $\mu \mathrm{m})$ and $T \approx 2 \%(T \approx 35 \%)$ for the rubbed (untreated) sample. We have calculated the twist coherence length $\xi_{t}$, which determines the width of the TN lines. Our analysis shows that the dominant parameter influencing $\xi_{t}$ is the cell thickness. The surface anchoring energy of the surrounding surface $W^{(a)}$ affects $\xi_{t}$ in the weak anchoring regime $\left(W^{(a)}\right.$ $<5 \times 10^{-6} \mathrm{~J} / \mathrm{m}^{2}$ ). In the uniaxial limit, the variation of $W^{(a)}$ can change $\xi_{t}$ for a factor of 2 at maximum. The simulations of the transmission intensity suggest that the anchoring strengths of the AFM line and the untreated polyimide are $W^{(\mathrm{AFM})} \approx 2 \times 10^{-5} \mathrm{~J} / \mathrm{m}^{2}$ and $W^{(\mathrm{NON})} \approx 5 \times 10^{-7} \mathrm{~J} / \mathrm{m}^{2}$, respectively.

These observations are in line with measurements of the effective azimuthal anchoring strength $W_{\phi}$ of the AFM scanned surface as a function of line separation $L$. With decreasing $L, W_{\phi}$ first increases proportional to $1 / L$ and saturates below $L=0.5 \mu \mathrm{m}$ at $W_{\phi} \approx 1.7 \times 10^{-5} \mathrm{~J} / \mathrm{m}^{2}$.

\section{ACKNOWLEDGMENTS}

Part of this work was supported by the EU Network SILC and by the Dutch Technology Foundation (STW). One of us (M.S.) acknowledges the NSRIM for the hospitality during his stay, and thanks E. Aret and S. Graswinckel of the group of Profesor Vlieg for their help with the experiments.

${ }^{1}$ A. J. Pidduck, G. P. Brayan-Brown, S. D. Haslam, and R. Bannister, Liq. Cryst. 21, 759 (1996).

${ }^{2}$ M. P. Mahajan and C. Rosenblatt, J. Appl. Phys. 83, 7649 (1998).

${ }^{3}$ M. Ruetschi, P. Grutter, J. Funfschiling, and H. J. Guntherodt, Science 256, 512 (1994).

${ }^{4}$ B. Wen and C. Rosenblatt, J. Appl. Phys. 89, 4747 (2001).

${ }^{5}$ M. Ruetschi, J. Funfschiling, and H. J. Guntherodt, J. Appl. Phys. 80, 3155 (1996).

${ }^{6}$ A. J. Pidduck, S. D. Haslam, G. P. Brayan-Brown, R. Bannister, and I. D. Kitely, Appl. Phys. Lett. 71, 2907 (1997).

${ }^{7}$ B. Wen, M. P. Mahajan, and C. Rosenblatt, Appl. Phys. Lett. 76, 1240 (2000).

${ }^{8}$ J. H. Kim, M. Yoneya, J. Yamamoto, and H. Yokoyama, Appl. Phys. Lett. 78, 3055 (2001).

${ }^{9}$ A. Rastegar, M. Skarabot, B. Blij, and T. Rasing, J. Appl. Phys. 89, 960 (2001).

${ }^{10}$ M. Behdani, A. Rastegar, S. H. Kashmiri, S. I. Missat, E. Vlieg, and T. Rasing, Appl. Phys. Lett. 80, 4635 (2002).

${ }^{11}$ S. Ruipeng, H. Ximin, J. Min, M. Kai, W. Zongkai, J. Hai, Y. Jianfeng, and J. Changfeng, Mol. Cryst. Liq. Cryst. 265, 335 (1995).

${ }^{12}$ P. G. de Gennes and J. Prost, The Physics of Liquid Crystals (Oxford University Press, Oxford, 1993).

${ }^{13}$ M. Nobili and G. Durand, Phys. Rev. A 46, R6174 (1992).

${ }^{14}$ F. J. Vesely, Computational Physics (Plenum, London, 1994).

${ }^{15}$ S. Chandrasekhar, Liquid Crystals (Cambridge University Press, UK, 1977). 